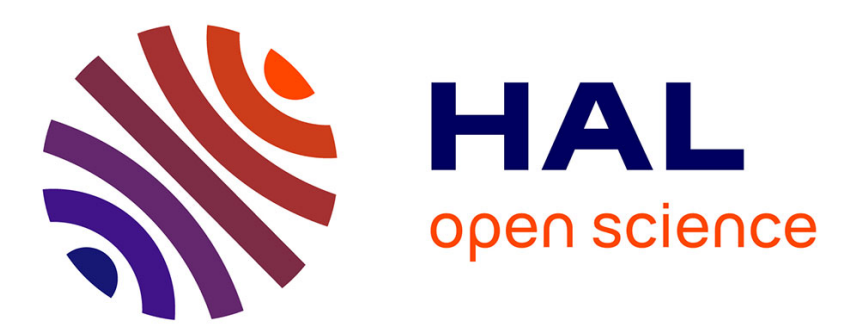

\title{
Online EV scheduling algorithms for adaptive charging networks with global peak constraints
}

Bahram Alinia, Mohammad Hajiesmaili, Zachary J Lee, Noel Crespi, Enrique Mallada

\section{- To cite this version:}

Bahram Alinia, Mohammad Hajiesmaili, Zachary J Lee, Noel Crespi, Enrique Mallada. Online EV scheduling algorithms for adaptive charging networks with global peak constraints. IEEE Transactions on Sustainable Computing, In press, pp.1-12. 10.1109/TSUSC.2020.2979854 • hal-02472860

\section{HAL Id: hal-02472860 https://hal.science/hal-02472860}

Submitted on 10 Feb 2020

HAL is a multi-disciplinary open access archive for the deposit and dissemination of scientific research documents, whether they are published or not. The documents may come from teaching and research institutions in France or abroad, or from public or private research centers.
L'archive ouverte pluridisciplinaire HAL, est destinée au dépôt et à la diffusion de documents scientifiques de niveau recherche, publiés ou non, émanant des établissements d'enseignement et de recherche français ou étrangers, des laboratoires publics ou privés. 


\title{
Online EV Scheduling Algorithms for Adaptive Charging Networks with Global Peak Constraints
}

\author{
Bahram Alinia, Mohammad H. Hajiesmaili, Zachary J. Lee, Noel Crespi, and Enrique Mallada
}

\begin{abstract}
This paper tackles online scheduling of electric vehicles (EVs) in an adaptive charging network (ACN) with local and global peak constraints. Given the aggregate charging demand of the EVs and the peak constraints of the ACN, it might be infeasible to fully charge all the EVs according to their charging demand. Two alternatives in such resource-limited scenarios are to maximize the social welfare by partially charging the EVs (fractional model) or selecting a subset of EVs and fully charge them (integral model). The technical challenge is the need for online solution design since in practical scenarios the scheduler has no or limited information of future arrivals in a time-coupled underlying problem. For the fractional model, we devise both offline and online algorithms. We prove that the offline algorithm is optimal. Using competitive ratio as the performance measure, we prove the online algorithm achieves a competitive ratio of 2 . The integral model, however, is more challenging since the underlying problem is strongly NP-hard due to $0 / 1$ selection criteria of EVs. Hence, efficient solution design is challenging even in offline setting. For offline setting, we devise a low-complexity primal-dual scheduling algorithm that achieves a bounded approximation ratio. Built upon the offline approximate algorithm, we propose an online algorithm and analyze its competitive ratio in special cases. Extensive trace-driven experimental results show that the performance of the proposed online algorithms is close to the offline optimum, and outperform the existing solutions.
\end{abstract}

Index Terms-Electric Vehicle, Online Scheduling, Approximation Algorithm, Competitive Analysis

\section{INTRODUCTION}

$\mathrm{T}$ O promote quick adoption of renewable energy sources, electrification of vehicles is a trend that has been globally advocated in recent years. According to a Bloomberg report, EVs will account for more than half of the new car sales by 2040 [1]. Consequently, it is expected that demand from EV charging will constitute a considerable portion of total demand. Currently, transportation consumes $29 \%$ of total energy in the US, while electricity production consumes $40 \%$ [2].

EV charging demand is a typical example of a deferrable load, and there is often considerable flexibility in the charging schedule. This property makes the problem of EV charging scheduling important and there is an extensive research along this direction in the literature (see the recent survey [3], and references therein). In most of the existing works, the EV charging demand is treated as a constraint to the problem in a low-load regime [4]. Motivated by rapid proliferation of EVs, this work tackles the EV scheduling in high-load regime, where given the aggregate charging demand of the EVs and the peak constraints of the charging

B. Alinia and N. Crespi are with the department of Networks and Mobile Multimedia Services (RS2M), Institut Mines-Telecom, Telecom SudParis, Evry, France (e-mail: bahram.alinia@telecom-sudparis.eu,noel.crespi@minestelecom.fr).

M. H. Hajiesmaili is with the College of Information and Computer Sciences, University of Massachusetts Amherst, Amherst, MA, USA (email: hajiesmaili@cs.umass.edu).

Z. J. Lee is with the Department of Electrical Engineering, California Institute of Technology, Pasadena, CA, USA (e-mail: zlee@caltech.edu). He is supported by the NSF Graduate Research Fellowship Program (Grant No. 1745301) and Resnick Sustainability Institute.

E. Mallada is with the Department of Electrical and Computer Engineering, Johns Hopkins University, Baltimore, MD, USA (e-mail: mallada@jhu.edu). network, it is not feasible to fully charge all the EVs according to their charging demand.

More specifically, this paper studies resourceconstrained EV charging scheduling in an adaptive charging network (ACN) governed by a single operator in a campus-scale location such as a university, a headquarters, etc. [5]. A notable example is the Caltech ACN [6], [7] where individual charging ports are organized into several charging stations (CSs) which are dispersed in a charging network with the capability of adaptive charging of the EVs. The problem is different from EV charging scheduling with capacity constraint in single station scenarios [2], [4], [8]-[13] (we refer to Section 2 for detailed discussions on related work), because of the essential need to respect the aggregate peak demand of the ACN. Note that, the ACN operator might limit the total power drawn from EVs to control costs [14], [15], reserve the capacity for other loads, and/or participate in demand-response programs.

We consider a scenario with multiple EVs where each EV has different charging profile in terms of availability, charging demand, charging rate limit, and valuation of getting charged (for details see Section 3.1.2). We formulate an online EV charging scheduling problem with the goal of selecting and scheduling a subset of EVs such that: (1) the charging demand of the selected EVs are (fully or partially) satisfied; (2) the charging rate limit of EV batteries are respected, (3) the global peak constraint of the ACN is satisfied [7]; (4) the local peak constraint of each CS is respected; and finally, (5) the total revenue obtained by the valuation of the EVs is maximized.

There are two main challenges in the design and implementation of scheduling algorithms for EVs satisfying the goals mentioned above. Firstly, the problem calls for online scheduling design. In practice, EVs arrive to the 
CS in online fashion and the scheduler has no information about the arrival and demand of the future EVs. Secondly, the underlying optimization problem in integral model is strongly NP-hard even in offline case (see Section 5). This is because the problem is a mixed integer linear problem and a "time-expanded" extension of knapsack problem which is known as a classic NP-hard problem. In this paper, we tackle the challenge of online design by following competitive algorithm design [16] and the challenge of NP-hardness by pursuing approximation algorithm design [17] and make the following contributions:

$\triangleright$ We first consider a fractional model (where EVs can be charged partially and the revenue is proportional accordingly) and design an optimal offline scheduling algorithm. We then develop an efficient online algorithm in which no exact or stochastic information about the future EV arrivals is given. Despite its simplicity, the algorithm is proved to be 2-competitive with optimal offline solution, i.e., the revenue of the proposed online algorithm is at least $1 / 2$ of the offline optimum, regardless of input sequence. Even though there are competitive algorithms in the literature for similar problems, to the best of our knowledge, our algorithm is the first 2-competitive algorithm which considers the charging rate limits.

$\triangleright$ We next study the more challenging scenario of the integral model, where EVs must receive all their demand to make revenue. We first propose a polynomial-time primaldual offline approximate algorithm. We analyze the approximation ratio of the algorithm and by strengthening the linear relaxed version of the mixed integer problem [18], we obtain an approximation ratio of $\alpha=1+\sum_{j=1}^{m} \frac{p_{j}}{p_{j}-q_{j}} \cdot \frac{s}{s-1}$, where $p_{j}$ is local peak constraint in station $j, q_{j}$ is the maximum charging rate of the EVs in station $j$ and $s$ is a slackness parameter. We highlight that when $p_{j} \gg q_{j}$ and $s$ is large enough, then $\alpha \approx m+1$, where $m$ is the number of stations in the ACN. Built on the basis of the offline algorithm, we devise an online algorithm, and discuss its competitive ratio in special cases.

$\triangleright$ We conduct a set of simulations to evaluate the performance of our proposed algorithms. The results of online algorithms for both integral and fractional settings are close to the optimum (within $90 \%$ and $94 \%$ for integral and fractional models in a representative scenario). In addition, our algorithm outperforms the existing scheduling algorithm in Caltech ACN [7] by 35\% for integral revenue model.

This paper represents follow-up work to our previous study [19], where we address a simplified version of the problems studied in this paper in fractional revenue model. The competitive analysis in [19] is done under the assumption that all EVs have the same maximum charging rate. Also, [19] does not study the integral revenue model. In this paper, we extend the results and propose a 2-competitive online algorithm with no assumption on the input parameters. Also, this paper investigates both fractional and integral models while considering global peak constraint and addressing multiple charging station scenario. Last, we consider more realistic trace-driven experiments in this paper and compare the results to an existing real-world scheduling algorithm.

\section{Related Work}

There is an extensive work in the broad topic of EV demand management and scheduling [3] such as as optimal operations with EV coordination and congestion management [20], [21], EV scheduling with incorporation of renewable energy, and energy storage systems [22], [23], and pricing and bidding [24]. We focus on the literature related to peak-constrained EV charging scheduling.

\subsubsection{Peak-Constrained EV Charging Scheduling}

There is an extensive literature on EV charging scheduling problem focusing on single station [4], [8], while the local and global peak constraints are omitted or only the local peak is considered. As we discuss in Section 3.2, the global optimal solution cannot be obtained by separately solving the single station problems. Hence, those solutions cannot be directly applied to the multiple station scenario with global peak constraints.

Studies in [25]-[30] tackled charging scheduling problem in multiple stations. The authors in [25] studied a global cost minimization EV charging scheduling problem, without taking into account the the maximum peak demand that the system can tolerate. [28]-[30] considered an offline multimicrogrid system with global peak constraint where each microgrid has a station and the goal is to manage electricity exchange between microgrids to minimize the operating costs. The authors assume that required information about EVs is available by forecast which may not represent a real scenario.

The authors in [26] use a similar model as in this paper, where both local and global peak constraints are considered. However, the authors solve the single-slot problem, which fails to provide a general solution taking into account EVs' arrival and departure times as considered in our study. Finally, as an alternative approach to control the peak [15], [31], some studies directly target minimizing the peak [2]. Although the peak is minimized in above works, it cannot guarantee that the minimized peak is tolerable by the ACN.

\subsubsection{Scheduling Under Demand Uncertainty}

A main challenge in EV scheduling problem is to cope with demand uncertainty. Many studies including [2], [4], [9][13], [32], [33] addressed online scheduling problem with different objectives, including social welfare, station revenue maximization, and maximum utilization of renewable sources.

Our problem in this paper is unique from above works in many respects. First, we study the problem in an ACN where several stations exist, while none of the above studies solve the problem under this setting. Second, the previous algorithms do not work for both integral and fractional charging models. In more relevant theoretical problems, [10], [11] put no limit on the charging rate of EVs which makes their solution impractical in real scenarios. Also, [2], [4], [9]-[11] do not consider the peak limit of the station. Finally, in [34], we considered a simplified EV charging scheduling in fractional model without global peak constraints and devised heuristic algorithms (without competitive and approximation analysis) with on-arrival commitment for EVs to notify the amount that they can receive by their departure. 


\subsubsection{Worst-case Analysis in Similar Scheduling Problems}

Similar underlying scheduling problems with slightly different settings have been studied in the literature in both offline and online settings. In offline setting, the problem is more interesting under integral revenue model where the problem becomes a combinatorial optimization problem and approximation algorithms have been used to find effective solutions. The performance of an approximation algorithm is determined by its approximation ratio for offline algorithms. On the other hand, competitive online algorithms are used in the online setting and competitive ratio is the performance metric which compares the algorithm's result to the offline optimal solution.

Offline integral model: Under integral revenue model, [35] proposes an offline algorithm for scheduling of batch jobs in cloud computing which is similar to EV scheduling problem. It is assumed that all jobs are available to be processed at time 0 . The authors propose a $\frac{C}{C-k} \cdot \frac{s}{s-1}$ approximation algorithm where $C$ is the cloud capacity and $s$ is the "slackness parameter" (see section 3.1.1 for the definition). Similarly, [36] tackles the offline integral problem and proposes a convex relaxation method to find a nearoptimal solution. No theoretical bound is provided for the algorithm and the performance is examined by simulation results. In this paper, we propose ICS for the EV scheduling problem with slightly different settings in the constraint sets, and tackle the problem in online setting and provide approximation analysis under several scenarios.

Online fractional model: In our recent study [19], we proposed two online algorithms referred to as WFAIR and WRAND for EV scheduling problem. The proposed algorithms provide a competitive ratio of $2-1 / U$ where $U$ is "scarcity level". However, the result only holds when all EVs have the same maximum charging rate. Also, [19] does not study the integral revenue model. In this paper, we propose FOCS as an online algorithm. Moreover, the current study investigates both fractional and integral revenue model while considering total peak constraint in the formulation and addressing multiple charging station scenario. In another work [37], two simple and natural online algorithms called FIRSTFIT and ENDFIT were developed and are proved to be 2-competitive. FOCS is extension of these algorithms by taking into account the maximum charging rate of the EVs and multiple charging station scenario (see "Remarks" at Section IV). As recent study, [38] provide online algorithms for EV scheduling problem, however, they do not take into account global peak constraint in the underlying problem.

Online integral model: Another direction is to tackle online scenario under integral revenue model, where the scheduling problem becomes fundamentally more challenging. We note that the integral scheduling problem is strongly NPHard even in the offline case [39]. Due to combined online and combinatorial challenges, there are very limited studies in this category. [40] provides an online algorithm without considering the maximum processing rate of the jobs where its competitive ratio can be arbitrary bad depending on "slackness" parameter. Our work extends this study for multiple station scenario and considering maximum charging rate for the EVs.

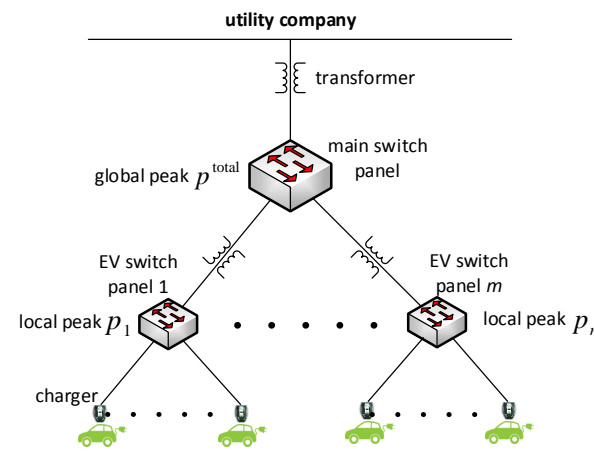

(a) Caltech adaptive charging network $(\mathrm{ACN})$ model with server

Fig. 1: System model [7].

\section{System Model and Problem Formulation}

\subsection{System Model}

We consider a time-slotted system model in which the time horizon is divided to $T$ equal length slots $t=\{1,2, \ldots, T\}$ (e.g., $T=24$ with time slots of 1 hour length).

\subsubsection{Charging Network}

Our charging network model is inspired by the Caltech ACN [7] as illustrated in Fig. 1a. In the Caltech EV charging network (located in a parking garage), electricity is distributed through a two-level transformer architecture from a main switch panel to multiple EV switch panels (2 panels in the current Caltech ACN). Each EV switch panel then is connected to several chargers $(\approx 25$ chargers per panel in the Caltech ACN). The total power drawn from the main switch panel by the charging network has a power limit of $p^{\text {total }}$, that is determined by the facility operator to control the costs, reserve the capacity for other loads, and/or participate in demand-response programs. In other words, $p^{\text {total }}$, which we refer to it as the global peak, hereafter, limits the maximum aggregate EV charging load at each time slot.

We assume that there are $m$ EV switch panels that represent $m$ CSs. In addition to the global peak constraint, each CS $j$ has a capacity constraint on its total power drawn, indicated by $p_{j}$, and referred to it as the local peak constraint. The value of $p_{j}$ is determined by the output power limit of the transformers installed between the main switch panel and EV switch panels and could be different for each EV switch panel. It is often observed that the charging demand of different CSs (EV switch panels in Fig. 1a) are well below the local peak constraints. To increase the flexibility due to heterogeneous charging demand of CSs, in the Caltech $\mathrm{ACN}$, the global peak constraint of the main switch can be over-provisioned, i.e., $p^{\text {total }}$ is less than the aggregate local peaks, $\left(\sum_{j} p_{j} \geq p^{\text {total }}\right)$. While this increases flexibility, it also couples the problem of EV charging scheduling across different CSs. Our solutions in this paper will be centralized ones which can be obtained through communication between the CSs and a central server as illustrated in Fig. $1 b$.

\subsubsection{EVs}

There are $n$ EVs in the system, indexed by $i$. EV $i$ is represented by a charging profile $\left\langle a_{i}, d_{i}, v_{i}, D_{i}, k_{i}\right\rangle$ indicating 
TABLE 1: Summary of key notations

\begin{tabular}{|cl|}
\hline Notation & Description \\
\hline \hline$T$ & Number of time slots, indexed by $t$ \\
$m$ & Number of CSs, indexed by $j$ \\
$n$ & Number of EVs, indexed by $i$ \\
\hline \hline$a_{i}$ & Arrival time of EV $i$ \\
$d_{i}$ & Departure time of EV $i$ \\
$D_{i}$ & Demand of EV $i$ \\
$v_{i}$ & Valuation of EV $i$ for receiving its demand $D_{i}$ \\
$k_{i}$ & Maximum charging rate of EV $i$ \\
$h(i)$ & CS of EV $i$ \\
\hline \hline$q_{j}$ & Maximum $k_{i}$ among all EVs in CS $j$ \\
$p_{j}$ & Maximum aggregate charging rate in station $j$ \\
$p^{\text {total }}$ & Maximum aggregate charging rate of all stations \\
\hline \hline$y_{i}^{t}$ & opt. variable, The amount that EV $i$ is charged at $t$ \\
\hline
\end{tabular}

its arrival time, departure time, willingness to pay, charging demand, and maximum charging rate, respectively. More specifically, the charging of EV $i$ can be scheduled within its availability window, $\left[a_{i}, d_{i}\right]$. The charging rate at each slot is bounded by $k_{i}$, a parameter that depends on the physical constraints of the battery and on-board charger. It is assumed that the charging profile of each EV is feasible with respect to its maximum charging rate and a slackness parameter $s \geq 1$, which is the minimum ratio between the park time of the EV and its minimum charging time, i.e., $D_{i} \leq k_{i}\left(d_{i}-a_{i}+1\right) / s$. The slackness parameter is imposed to tune the flexibility of the charging scheduling. In extreme case $s=1$, the flexibility is minimum and the flexibility improves as $s$ increases. We assume that EV owners select their CS, perhaps the nearest to them, and so the assignments are given to the problem. Define $h(i)$ as the CS of EV $i$. Moreover, $q_{j}$ denotes the maximum $k_{i}$ among all EVs in CS $j$, i.e., $q_{j}=\max _{h(i)=j} k_{i}$. Finally, $v_{i}$ is the willingness to pay of EV $i$ to receive its entire demand $D_{i}$ before the departure time $d_{i}$. Note that in resource-constrained EV scheduling in $\mathrm{ACN}$, it is not feasible to fulfill the entire demand of all EVs, hence, the problem turns into a resource allocation one with the goal of maximizing the aggregate value (a.k.a. utility) obtained from the EVs. In this way, each user can announce $v_{i}$ as its willingness to pay to get charged.

\subsubsection{Revenue Models}

We consider two revenue models: (i) Fractional revenue model: In this model, the fractional charging is allowed, i.e., the revenue from each $\mathrm{EV}$ is proportional to the fraction of the demand that is fulfilled [12] (see Eq. (2)). We tackle this model in Section 4. (ii) Integral revenue model: In this model, EV $i$ pays $v_{i}$ if it is fully charged and zero otherwise, i.e., there is no partial revenue for partial charging. This is the model that is considered in [9], [10]. We tackle this model in Section 5 .

\subsection{Problem Formulation}

We formulate an optimization problem to schedule the charging of the EVs with the objective of maximizing total revenue obtained from charged EVs while respecting local and global peak constraints. Note that each revenue model makes the underlying optimization problem fundamentally different. More specifically, the fractional charging model is a linear problem. Integral revenue model, however, turns the underlying charging scheduling problem to a Mixed Integer Linear Program (MILP). The integer nature originates from the 0/1-selection decision on EVs. We formulate Scheduling Problem for Adaptive charging Network (SPAN) under fractional revenue model as follows:

$$
\begin{aligned}
\text { SPAN : } \max & \sum_{i=1}^{n} \frac{v_{i}}{D_{i}} \sum_{t=a_{i}}^{d_{i}} y_{i}^{t} \\
\text { s.t. } \quad & \sum_{t=a_{i}}^{d_{i}} y_{i}^{t} \leq D_{i}, \quad \forall i, \\
& \sum_{i=1}^{n} y_{i}^{t} \leq p^{\text {total }}, \quad \forall t, \\
& \sum_{i: h(i)=j} y_{i}^{t} \leq p_{j}, \quad \forall t, j, \\
& y_{i}^{t} \leq \frac{k_{i}}{D_{i}} \sum_{t^{\prime}=a_{i}}^{d_{i}} y_{i}^{t^{\prime}}, \quad \forall i, t, \\
\text { vars. } \quad & y_{i}^{t} \geq 0, \quad \forall i, t,
\end{aligned}
$$

where $y_{i}^{t}$ is the amount that EV $i$ is charged at slot $t$. Constraint (1a) ensures that the aggregate amount received by EV $i$ is at most the demand $D_{i}$. The global and local peak constraints are represented by constraints (1b) and (1c), respectively.

The constraint (1d) enforces the maximum charging rate of EVs. The straightforward way to express this constraint is to simply state that at each time slot $t$, the charging rate of EV $i$ should be less than or equal to its maximum charging rate, i.e., $y_{i}^{t} \leq k_{i}, \forall i, t$. However, for the sake of effective algorithm design for integral model and reducing the integrality gap of the relaxed linear problem, this constraint is strengthened in the form of Eq. (1d). Note that in case that the aggregated charging of EV $i$ during its availability window is equal to its demand, i.e., $\sum_{t^{\prime}=a_{i}}^{d_{i}} y_{i}^{t^{\prime}}=D_{i}$, Eq. (1d) reduces to the simple form of $y_{i}^{t} \leq k_{i}$. This is a natural way in approximation algorithm design to improve the performance of the algorithms under linear-relaxation based design [18].

The SPAN is an extension of formulated problem in [35] where a job scheduling problem in cloud applications is studied. It turns out that the resource allocation problem in cloud systems and the EV charging scheduling problem in a single station share similar structure. Indeed, each charging profile in our scheduling problem can be seen as a job in cloud system with a deadline, value, and CPU demand. The SPAN, however, comes with an additional constraint (1b), which makes it different from the problem in [35], such that the existing solution will not work in the new setting. More importantly, this paper studies both offline and online solutions for the problem in both fractional and integral settings, while [35] tackles only the offline integral model.

\section{Fractional Revenue Model}

In the fractional model, the revenue of the CS from EV $i$ is directly proportional to the amount of resource that the EV is received, i.e.,

$$
v_{i}^{\mathrm{f}}=\frac{\sum_{t} y_{i}^{t}}{D_{i}} v_{i},
$$

where $v_{i}$ is the gain, if the entire demand $D_{i}$ is fulfilled and $v_{i}^{\mathrm{f}}$ is the fractional gain. We propose a simple algorithm, called FCS, with low computational complexity of 
TABLE 2: Proposed algorithms and their properties.

\begin{tabular}{|l|c|c|c|c|}
\hline Algorithm & Revenue model & Type & Optimality & Complexity \\
\hline \hline FCS & fractional & offline & Optimal & $O\left(n^{2} T+n T^{2}\right)$ \\
\hline ICS & integral & offline & $\left(1+\sum_{j=1}^{m} \frac{p_{j}}{p_{j}-q_{j}} \cdot \frac{s}{s-1}\right)$-approximate & $O\left(n T \log T+n^{2} T\right)$ \\
\hline FOCS & fractional & online & 2 -competitive & $O\left(n^{2} T\right)$ \\
\hline IOCS & integral & online & $b\left(1+\frac{p}{p-q} \cdot \frac{s}{s-1}\right)$-competitive, $(m=1)$ & $O\left(n^{2} T\right)$ \\
\hline
\end{tabular}

$O\left(n^{2} T+n T^{2}\right)$ for the fractional model that finds the optimal solution in offline setting. Note that even though linear programs can be solved in polynomial time in general, the complexity of our proposed algorithm is much lower than the general linear program algorithms. Moreover, our proposed offline algorithm applies a valley-filling strategy to reduce the peak. A summary of proposed algorithms with their complexity is given in Table 2 .

\subsection{Optimal Offline Design}

In this section, we propose an optimal algorithm for offline fractional model. As a natural solution, one may think of a greedy algorithm as follows: at each time slot, charge the $\mathrm{EV}(\mathrm{s})$ with highest unit-value and process others if only the remaining resource cannot be allocated to the selected $\mathrm{EV}(\mathrm{s})$ (e.g., due to maximum charging rate constraint or fulfillment of the EVs' demand). This approach is a popular, yet straightforward method in the scheduling. However, it turns out that this approach cannot provide an optimal solution to the problem even in fractional revenue model. In fact, this solution is only 2-approximation (i.e., the profit obtained by the algorithm would be $1 / 2$ of the optimum). As an intuition of the proof think of two EVs in a single charging station with power capacity $p$ and $\frac{v_{1}}{D_{1}}-\frac{v_{2}}{D_{2}}=\epsilon$ where $\epsilon>0$ is an arbitrary small number, $a_{1}=a_{2}=1, d_{1}=2, d_{2}=1$ and $D_{1}=D_{2}=k_{1}=k_{2}=p$. Then, applying the simple greedy, EV 1 will be selected at time slot 1 and EV 2 will have no chance to get charged due to its deadline constraint while EV 1 could wait until time slot 2 and still receive all its demand.

We refer the proposed algorithm in this secion as the FCS and summarize it as Algorithm 1. The FCS works in two phases. In the first phase (Section 4.1.1), the algorithm decides on the amount of resource to be allocated to each EV within its availability window and reserves resources accordingly. In this phase, the details of allocation is not known. The actual resource allocation is done in the second phase (Section 4.1.2) by setting variables $y_{i}^{t}$.

Before discussing the details of the algorithm, we give formally define the notion of "super interval" to facilitate our algorithm design.

Definition 1. Time interval $\left[\delta, \delta^{\prime}\right]$ is a "super interval" for interval $\left[t, t^{\prime}\right]$ if $1 \leq \delta \leq t$ AND $t^{\prime} \leq \delta^{\prime} \leq T$. Moreover, $\mathcal{I}_{t, t^{\prime}}$ is the set of all super intervals of interval $I_{t, t^{\prime}}$ i.e., $\mathcal{I}_{t, t^{\prime}}=\left\{\left[\delta, \delta^{\prime}\right]: 1 \leq \delta \leq t A N D t^{\prime} \leq \delta^{\prime} \leq T\right\}$.

The number of super intervals of an interval is at most $T^{2}$ and at least one (for interval $[1, T]$ ).

Let $R_{i}$ be the amount of resource that is reserved for EV $i$ by the FCS and $I_{t, t^{\prime}}$ as time interval $\left[t, t^{\prime}\right]$. Then, assuming that charging demands are sorted in non-increasing order of their unit values, $A_{j}^{i}\left(t, t^{\prime}\right)$ is the aggregate residual resource

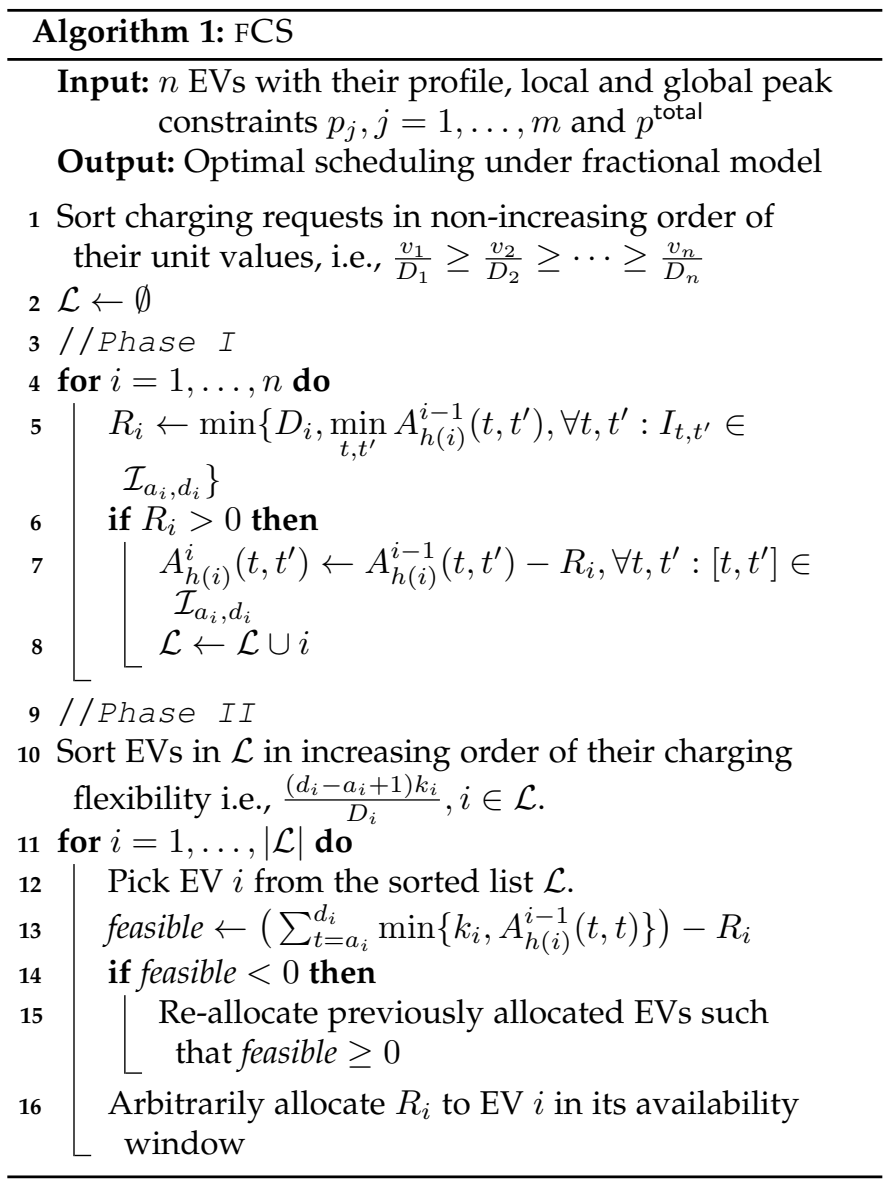

in interval $I_{t, t^{\prime}}$ at station $j$ assuming that the reservation for EVs 1 to $i$ is accomplished. We now explain in detail each phase of the algorithm.

\subsubsection{Phase I-Reservation}

In Line 1, the EVs are sorted in a non-increasing order of their unit values. In Line 5, the FCS processes demand of $\mathrm{EV} i$, picked from top of the ordered list, and sets $R_{i}$ as the amount to be reserved for EV $i$ which will be allocated in Phase II. In Line 7, the residual resource of all intervals in set $\mathcal{I}_{a_{i}, d_{i}}$ decreases by $R_{i}$ and EV $i$ is added to the set of selected EVs.

Lemma 1. Provided that for EV $i$ we have

$$
R_{i} \leq \min \left\{D_{i}, \min _{t, t^{\prime}} A_{j}^{i-1}\left(t, t^{\prime}\right), \forall t, t^{\prime}: I_{t, t^{\prime}} \in \mathcal{I}_{a_{i}, d_{i}}\right\}
$$

with a dummy $A_{j}^{0}\left(t, t^{\prime}\right)$ defined as $A_{j}^{0}\left(t, t^{\prime}\right)=\left(t^{\prime}-t+1\right) \times$ $\min \left\{p^{\text {total }}, p_{j}\right\}$, then there is a feasible allocation to allocate $R_{i}$ to $E V i$ in its availability window $\left[a_{i}, d_{i}\right]$.

In Lemma $1, A_{j}^{0}\left(t, t^{\prime}\right)$ indicates the available resource when no charging request is processed in $I_{t, t^{\prime}}$. In Eq. (3), the 
second term, i.e., $\min _{t, t^{\prime}} A_{j}^{i-1}\left(t, t^{\prime}\right)$, indicates the minimum remaining resource in all super intervals of interval $I_{a_{i}, d_{i}}$. For $i \geq 1, A_{j}^{i}\left(a_{i}, d_{i}\right)$ is defined as follows:

$$
A_{j}^{i}\left(a_{i}, d_{i}\right)= \begin{cases}A_{j}^{i-1}\left(a_{i}, d_{i}\right)-R_{i} & j=h(i), \\ A_{j}^{i-1}\left(a_{i}, d_{i}\right) & j \neq h(i) .\end{cases}
$$

The optimal value of $R_{i}, i=1, \ldots, n$ is set according to the following lemma:

Lemma 2. Given $n E V$ s sorted in a non-increasing order of the unit values, $v_{1} / D_{1} \geq v_{2} / D_{2} \geq \cdots \geq v_{n} / D_{n}$, and the value of $R_{i}$, where $R_{i}$ is set after $R_{i-1}, i=2, \ldots, n$ by Eq. (3), then,

$$
R_{i}=\min \left\{D_{i}, \min _{t, t^{\prime}} A_{h(i)}^{i-1}\left(t, t^{\prime}\right), \forall t, t^{\prime}: I_{t, t^{\prime}} \in \mathcal{I}_{a_{i}, d_{i}}\right\}, \forall i,
$$

is the optimal value for $R_{i}$.

\subsubsection{Phase II- Allocation}

Lemma 1 shows that there is a feasible scheduling to allocate the reserved resources. However, despite its feasibility, it is not straightforward to find such a schedule. For example, assume that for $\mathrm{EV} i, R_{i}=10$ and $k_{i}=4$. It is possible that all available resources are concentrated in a single time slot but $\mathrm{EV} i$ cannot use more than $4 \mathrm{kWh}$ of it. In this situation, the previously allocated resources in interval $I_{a_{i}, d_{i}}$ should be reallocated such that the concentrated resources are dispersed and we have $\sum_{t=a_{i}}^{d_{i}} \sigma_{t} \geq R_{i}$ where $\sigma_{t}=\min \left\{k_{i}, A_{h(i)}^{i-1}(t, t)\right\}$ is the maximum resource that can be allocated to EV $i$ at time slot $t$. Since the total amount of allocated resource does not change in the interval, such dispersion is possible and can be done by a simple algorithm in which allocates $\min \left\{k_{i}, A_{h(i)}^{i-1}(t, t)\right\}$ starting from time slot $t=a_{i}$ until $R_{i}$ units is allocated. To further reduce the peak of the system, we will develop SMARTALLOCATE algorithm (See Section 5) which acts more intelligent so that Line 16 of the FCS can be replaced by "Run SMARTAllocate $\left(i, R_{i}\right)$ ".

Theorem 1. FCS is an optimal solution under fractional revenue model. FCS.

The following theorem characterizes the complexity of

Theorem 2. The time complexity of FCS algorithm is $O\left(n^{2} T+\right.$ $\left.n T^{2}\right)$ where $n$ is the number of EVs and $T$ is number of time slots.

\subsection{Online Scenario}

In this section, we devise an algorithm for the scenario that EVs arrive in online fashion. The scheduling decisions at each time slot are made given the information of available EVs and neither exact values nor stochastic modeling of future arrivals is available. Our goal is to obtain a competitive ratio for the online algorithm. A scheduling algorithm $\mathcal{A}$ is $c$-competitive for $c \geq 1$ if the revenue obtained by the optimal offline algorithm is at most $c$ times the algorithm $\mathcal{A}^{\prime}$ 's revenue for any input sequence [16].

The proposed online algorithm for the fractional model, referred to as FOCS, is listed as Algorithm 2. The FOCS is a simple yet efficient algorithm that always selects EVs with highest unit value to allocate as follows. First, the algorithm sorts the available EVs at each time slot $t$ based on their unit value (Line 2). In this step, the algorithm breaks ties based on EVs' deadline i.e., if two EVs have the same unit value, then the one with earliest deadline comes first in the sorted list. Next, the FOCS selects one EV at a time from the sorted list to allocate with maximum charging rate considering the EV's remaining demand, maximum charging rate, and peak constraints (Lines 3-4). The allocation is continued until all resources are allocated or there is no more EV that could be allocated. The time complexity of the FOCS is $O\left(n^{2} T\right)$, determined by cost of its "for" loop multiplied by total number of times that the algorithm needs to be run.

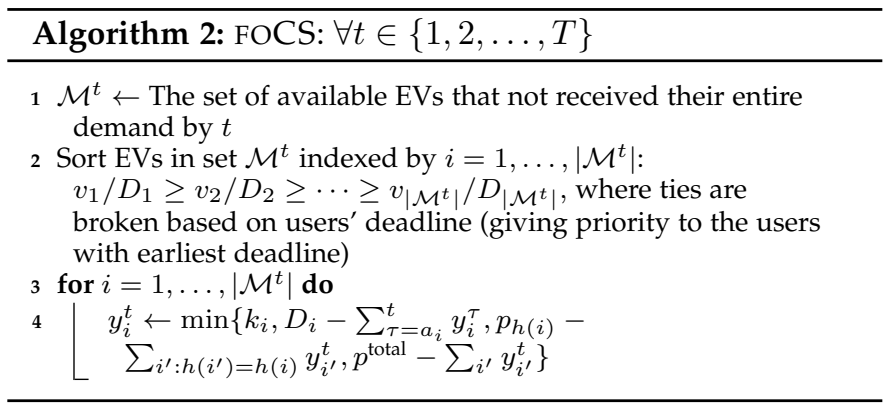

Despite the simplicity of FOCS which makes it easy to implement, its performance is sound and within a constant factor of the offline optimum. We now proceed to analyze the performance of the FOCS by first giving some preliminaries.

Fix an optimal scheduling and let $\mathcal{S}_{\mathrm{FOCS}, t}$ and $\mathcal{S}_{\mathrm{OPT}, t}$ be the sets of EVs selected by the FOCS and optimal solution at time slot $t$, respectively. Let $y_{i}^{t}$ and $z_{i}^{t}$ be the charging rate of EV $i$ set by FOCS and OPT, respectively. We define $\Delta_{i}^{t}$ as follows:

$$
\Delta_{i}^{t}= \begin{cases}\min \left\{z_{i}^{t}-y_{i}^{t}, r_{i, t}\right\} & i \in \mathcal{S}_{\mathrm{OPT}, t}, z_{i}^{t}>y_{i}^{t}, \\ 0 & \text { otherwise, }\end{cases}
$$

where $r_{i, t}$ is the remaining demand of EV $i$ by the end of time slot $t . \Delta_{i}^{t}>0$ indicates that the optimal algorithm allocated $\Delta_{i}^{t}$ units more resources to EV $i$ than the FOCS by time slot $t$ that could be feasibly allocated by FOCS to the $\mathrm{EV} i$. If for any EV $i \in \mathcal{S}_{\mathrm{OPT}, t}$ and time slot $t \in \mathcal{T}$ we have $y_{i}^{t}=z_{i}^{t}$, i.e., $\Delta_{i}^{t}=0$, then the FOCS is obviously optimal because it gains whatever the optimal solution gains. We define loss of the FOCS imposed by EV $i$ as follows:

$$
l_{i, t}=\Delta_{i}^{t} \frac{v_{i}}{D_{i}},
$$

Note that the loss always takes a non-negative value as $\Delta_{i}^{t} \geq 0$. When FOCS sets charging rate of an EV less than its rate in the optimal solution, it gains $\Delta_{i}^{t} v_{i} / D_{i}$ less than the optimal solution from that EV. An upper bound for the distance between the optimal objective value (denote by OPT) and the revenue of the FOCS (denote by ALG) is summation of the losses over all time slots and all EVs, i.e.,

$$
\mathrm{OPT}-\mathrm{ALG} \leq \sum_{t=1}^{T} \sum_{i \in \mathcal{S}_{\mathrm{OPT}, t}} l_{i, t} .
$$

In addition to the amount of loss, the gain of the algorithm from charging alternative EVs should also be taken into account in comparison between OPT and ALG. 
Let $i \in \mathcal{S}_{\mathrm{OPT}, t}, i \notin \mathcal{S}_{\mathrm{ALG}, t}$ and $g_{i, t}$ be the gain that the FOCS obtains from charging another EV instead of $i$ at time slot $t$. We are going to show that in the FOCS, for each EV $i$ with $l_{i, t}>0$ there must be another EV (denote by $i^{\prime}$ ) where $y_{i^{\prime}}^{t} \geq \Delta_{i}^{t}$ and $\frac{v_{i^{\prime}}}{D_{i^{\prime}}} \geq \frac{v_{i}}{D_{i}}$ which means in resource allocation phase for EV $i$, the FOCS allocated the difference $\Delta_{i}^{t}$ to another EV with the same or higher unit value. This can be proved by considering the fact that (i) the selected EVs have higher unit values than the unselected EVs, and (ii) the charging rate of the selected EVs are set to the maximum feasible value. Moreover, the FOCS is "work-conserving" i.e., it does not let any resource remain unused if there are some EVs that can use it.

Let $g_{i, t}$ denote the gain that FOCS obtains from allocating the same amount of resource that optimal algorithm allocated to EV $i$ (with size $z_{i}^{t}$ ) to another $\mathrm{EV}\left(\mathrm{s}\right.$ ). If $\Delta_{i}^{t}=0$, the loss is zero. If $\Delta_{i}^{t}>0$, then by the charging strategy that the FOCS uses we can conclude that (i) $i$ is not finished by the FOCS, and (ii) no more resources from station $p_{h(i)}$ can be feasibly allocated, otherwise, the FOCS could allocate more resources to EV $i$. Therefore, the $\Delta_{i}^{t}$ units of the resource are allocated to one or multiple other EVs (denote them by set $\mathcal{J}_{i}^{t}$ ) by the FOCS. Moreover, it must hold that all the EVs in set $\mathcal{J}_{i}^{t}$ have a unit value equal to or higher than $v_{i} / D_{i}$ which yields $g_{i, t} \geq l_{i, t}$, otherwise, the FOCS should not prefer the EVs in $\mathcal{J}_{i}^{t}$ to $i$. Since the result holds for any arbitrarily EV $i$, we can get the following:

$$
\sum_{i=1}^{n} \sum_{t=1}^{T} l_{i, t} \leq \sum_{i=1}^{n} \sum_{t=1}^{T} g_{i, t} .
$$

Moreover, the total gain of FOCS, i.e., ALG, is equal to sum of its gains from each single EV:

$$
\mathrm{ALG}=\sum_{i=1}^{n} \sum_{t=1}^{T} g_{i, t}
$$

With the above discussion and using Eqs. (6), (7) and (8) we are able to derive a competitive ratio of 2 for the FOCS.

Theorem 3. The FOCS is 2-competitive.

Remarks: When there is only one CS and EVs have no limit on their charging rate, the FOCS is identical to the FIRSTFIT algorithm [37] which is known to be 2-competitive for classic job scheduling problem. However, the charging rate limitation is crucial for EV charging problem. Moreover, [37] uses a "charging argument" to prove the competitive ratio of the proposed algorithm which cannot be directly applied to our problem. Thus, the FOCS extends the FIRSTFIT and makes it practical for the EV charging scenario. Moreover, the proof technique used for the competitive analysis of the FOCS is fundamentally different from the one used in [37].

\section{InTEgral ReVenue Model}

The MILP form of the SPAN in integral model is a generalized form of the 0/1-knapsack problem which is a wellknown NP-hard problem. To give an intuition, consider the scheduling problem in a single time slot (i.e, $T=1$ ). Then, allocating power resources to the EVs is equivalent to allocating the capacity of knapsack to the items. In Section 5.1, we propose a fast polynomial time offline approximation algorithm for the integral problem. In Section 5.2, we extend the result and propose an online algorithm for the integral model.

\subsection{Offline Scenario}

We design our offline scheduling algorithm under integral model referred to as ICS, to solve the SPAN approximately. Since the performance analysis of the proposed algorithm relies on a dual fitting method and utilizes weak duality property, we first need to construct the dual problem of SPAN. Toward this, we introduce variables $\alpha, \beta, \gamma$ and $\pi$. Generally, in primal-dual approximation algorithm design, each constraint (resp. variable) in primal (resp. dual) problem is associated with a variable (resp. constraint) in dual (resp. primal) problem. In our case, constraints (1a) (1b), (1c) and (1d) in the SPAN are respectively associated with dual variables $\alpha, \beta, \gamma$ and $\pi$ (for more details see [17]). The dual problem is formulated as follows:

$$
\begin{array}{ll}
\min & \sum_{i=1}^{n} D_{i} \alpha_{i}+\sum_{j=1}^{m} \sum_{t=1}^{T} p_{j} \beta(t)+\sum_{t=1}^{T} p^{\text {total }} \gamma(t) \\
\text { s.t. } & \alpha_{i}+\beta(t)+\gamma_{i}+\pi(t)-\frac{k_{i}}{D_{i}} \sum_{t^{\prime}=a_{i}}^{d_{i}} \pi_{i}\left(t^{\prime}\right) \geq \frac{v_{i}}{D_{i}} \\
& \forall i, t \in\left[a_{i}, d_{i}\right] \\
\text { vars. } & \alpha_{i}, \beta_{i}, \gamma, \pi_{i}(t) \geq 0, \quad \forall i, t .
\end{array}
$$

\subsubsection{Explanation of the Main Algorithm}

Our algorithm design is inspired by the basic algorithm proposed in [35]. The algorithm in [35], however, works for a single CS where arrival time of all EVs are the same and there is no global peak constraint. The ICS algorithm (listed as Algorithm 3) works in two phases. In the first phase it sorts the charging requests based on their unit values in a non-increasing order. Then, it selects most valuable demand. If the remaining resource is enough to cover the entire demand of the $\mathrm{EV}$, it is admitted to receive the demand (Lines 6-7).

Scheduling of the Selected EV: If the feasibility check passed (Line 6 in Algorithm 3), ICS calls sub-procedure SMARTALLOCATE to allocate required resources in interval $\left[a_{i}, d_{i}\right]$. Then, $\alpha_{i}$ is set to $v_{i} / D_{i}$ in order to cover dual constraint in Eq. (9a) (Lines 7-8).

In Smartallocate, let us define $W(t, h(i))=$ $\sum_{i^{\prime}: h\left(i^{\prime}\right)=h(i)} y_{i^{\prime}}^{t}$ as total workload at time slot $t$ in CS $h(i)$ and $\bar{W}(t, h(i))$ as total available load to allocate at time slot $t$ for CS $h(i)$. We always have $\bar{W}(t, h(i))+W(t, h(i))=p_{h(i)}, \forall t, i$. For scheduling, SMARTALLOCATE applies two main policies: 1) valley-filling and, 2) right-to-left allocation. With valley-filling, the slots with more available resources are preferred which helps to reduce the peak of the system. Right-to-left allocation is used when two or more time slots are equal in terms of their remaining resources. A ranking based approach is used to apply the aforementioned policies. To charge EV $i$, SMARTALlOCATE ranks time slots in interval $\left[a_{i}, d_{i}\right]$. Then, charging is done by allocating resources from the higher ranked time slot to lowest one. The rank of a time slot $t$ 
is calculated based on remaining resources in the time slot (valley-filling) and value of $t$ (right-to-left allocation).

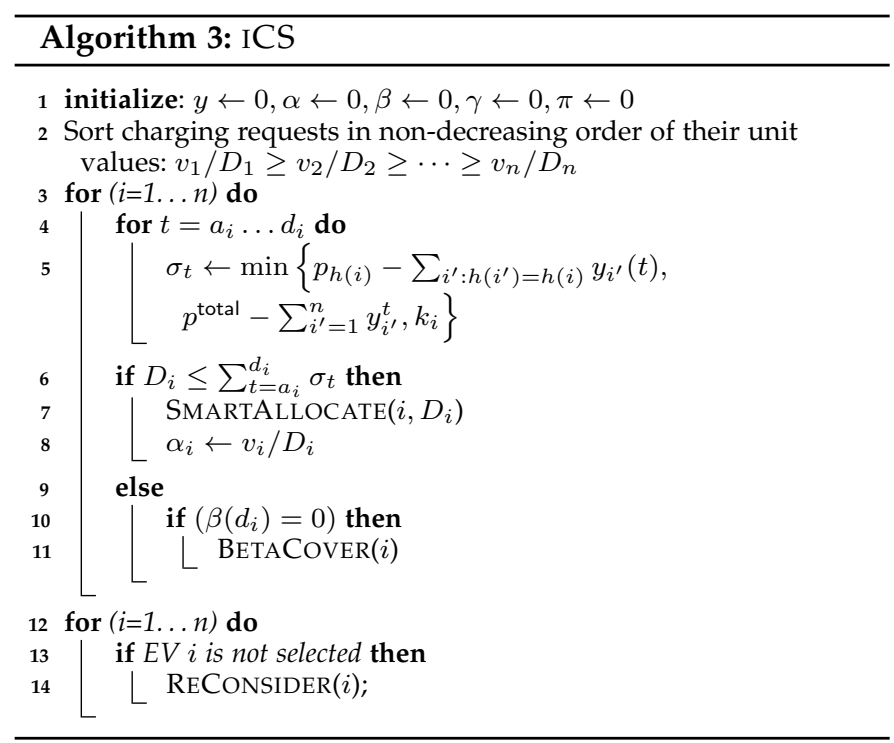

Dual Feasibility of the Non-selected EVs: If remaining power resource is not enough to fully charge $\mathrm{EV} i$, i.e., $D_{i}>\sum_{t=a_{i}}^{d_{i}} \sigma_{t}$, the EV cannot be selected. However, we still need to satisfy constraint (9a) in dual problem which is done by calling BETACOVER $(i)$. To cover the constraint (9a) for EV $i$, sum of dual variables for all $t \in\left[a_{i}, d_{i}\right]$ should be greater than or equal to $v_{i} / D_{i}$. $\operatorname{BETACOVER}(i)$ sets $\beta(t)$ to $v_{i} / D_{i}$ for all time slots $t$ in interval $\left[t_{\text {cov }}, R\left(d_{i}\right)\right]$ (Lines 3-4 of Algorithm 5). Observe that $\beta\left(t^{\prime}\right) \geq v_{i} / D_{i}, \forall t^{\prime}<t_{\text {cov }}$ (with $t_{\text {cov }}>1$ ) considering that the demands are sorted in a nonincreasing order of the unit-values and $\beta\left(t^{\prime}\right)$ is already set to $v_{i^{\prime}} / D_{i^{\prime}}$ when processing the earlier charging demand of $\mathrm{EV} i^{\prime}$ which is not selected. Hence, $v_{i^{\prime}} / D_{i^{\prime}} \geq v_{i} / D_{i}$, thereby $\beta(t) \geq v_{i} / D_{i}$, and the dual constraint in (9a) is satisfied. Lines 1-4 of BETACOVER is enough to cover the dual constraint. However, the algorithm continues in Lines $5-8$ by setting a variable $\Phi_{i^{\prime}}(t)$ for time slots $t=1, \ldots, R\left(d_{i}\right)$ to a value dependent to amount of the resource that a selected EV $i^{\prime}$ received at slot $t . \Phi_{i^{\prime}}(t)$ will be used in approximation analysis of the main algorithm and has no effect on the scheduling of EVs.

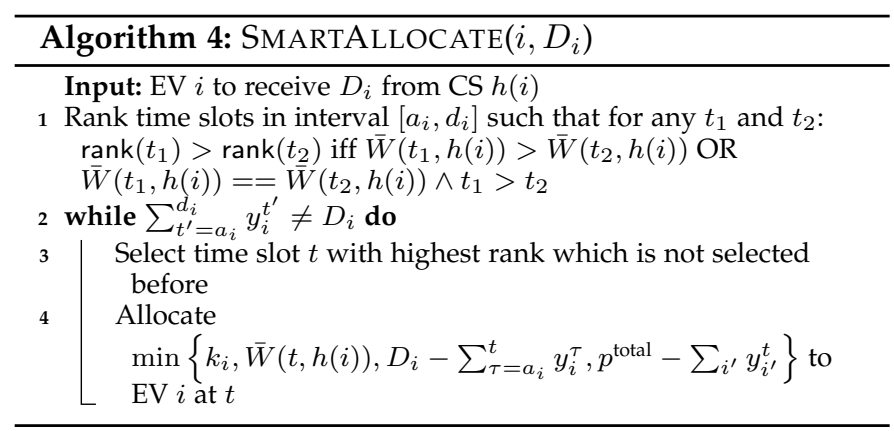

Improving the Gain: In the second phase, the ICS tries to increase total value of selected EVs by calling RECON$\operatorname{SIDER}(i)$ on every unselected EV $i$ (Lines 12-14). The RECONSIDER(i) (listed as Algorithm 6) checks that whether the total revenue can be increased by replacing some selected
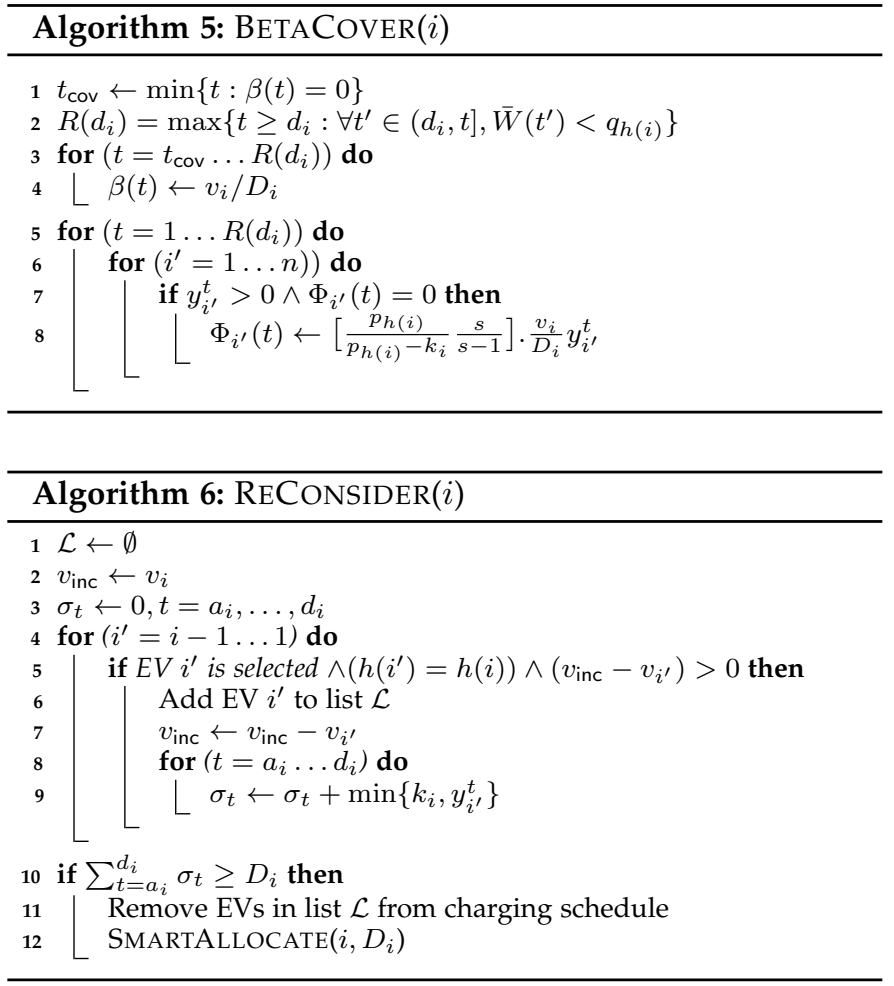

EVs with EV $i$ or not (Lines 4-9). If such EVs are found, the algorithm stops their charging and allocates EV $i$ using SMARTAlLOCATE (Lines $10-12$ ).

\subsubsection{Analysis}

In a primal-dual algorithm, the goal is to design an algorithm in a way that it produces a good solution for primal problem (with primal value $\Gamma$ ) and a feasible solution for the dual problem (with dual value $\Lambda$ ). Then, assuming that the primal problem is a maximization problem, to prove that the algorithm is $c$-approximation (for $c \geq 1$ ), the important part is to show that $\Lambda \leq c \Gamma$. Then, based on weak duality theorem we have $\Lambda \geq \mathrm{OPT}$, and it is concluded that $\Gamma \geq \frac{1}{c} \times$ OPT where OPT is the optimal value. Based on the above understanding, the following theorem scrutinizes the approximation ratio of the ICS assuming that arrival times are the same for all EVs.

Theorem 4. ICS algorithm is a $\left(1+\sum_{j=1}^{m} \frac{p_{j}}{p_{j}-q_{j}} \cdot \frac{s}{s-1}\right)$ approximation when EVs have same arrival time.

Note that in the case that the system is flexible enough, i.e., $s \gg 1$, and the maximum charging rates of stations are much bigger than those of EVs, i.e., $p_{j} \gg q_{j}, \forall j$, the approximation ratio approaches $m+1$. And in the case that there is one single station, the approximation ratio is 2 .

\subsection{Online Scenario}

Due to the binary selection variable, the online solution design under integral model is more challenging than the one for fractional model. We propose IOCS that is built upon the offline ICS. In particular, the IOCS calls the ICS at each time slot for the set of available EVs, however, any algorithm that is designed for offline integral model can 
be used alternatively. Hereinafter, $\mathcal{A}$ refers to the ICS or a similar algorithm.

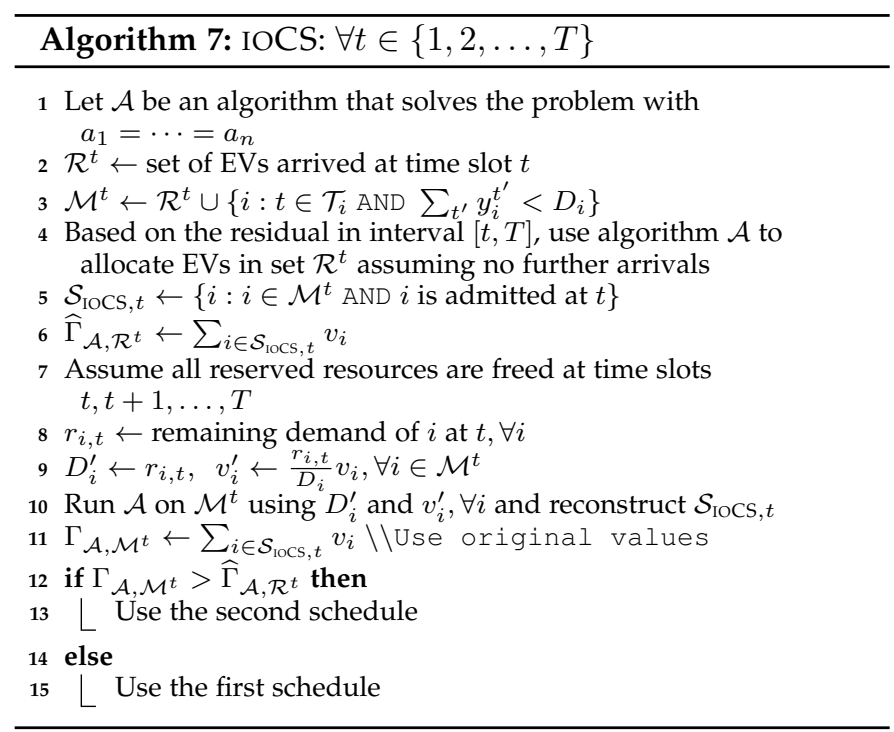

The IOCS is summarized as Algorithm 7. At slot $t$, the IOCS compares two scheduling results returned by $\mathcal{A}$ and chooses among them. In the first scheduling, the IOCS keeps all reserved resources in interval $[t, T]$ intact. Then, for utilizing the remaining resources, the algorithm runs $\mathcal{A}$ over arrived EVs at time slot $t$. In this case, the total revenue obtained by the active EVs (i.e., EVs that are available but not received their entire demand yet) is denoted by $\widehat{\Gamma}_{\mathcal{A}, \mathcal{R}^{t}}$ (Line 6 of the algorithm). In the second scheduling, the IOCS considers the case that it can sacrifice the previously admitted EVs by canceling their reservations and allocating the freed resources to more valuable demands. For this purpose, the algorithm modifies the demand and valuation of the previously admitted EVs such that each demand is replaced by the EV's remaining demand, and the valuation of the EV is proportionally calculated based on the remaining demand (Line 9 of the algorithm) so that the unit values of EVs do not change. Then, the IOCS runs $\mathcal{A}$ on set of active EVs $\mathcal{M}^{t}$ where the corresponding gain is denoted by $\Gamma_{\mathcal{A}, \mathcal{M}^{t}}$. If $\Gamma_{\mathcal{A}, \mathcal{M}^{t}}>\widehat{\Gamma}_{\mathcal{A}, \mathcal{R}^{t}}$, the IOCS forgets the previously admitted EVs and follows the second scheduling.

The following theorem characterizes the competitive ratio of the IOCS in a special case.

Theorem 5. Let $\mathcal{A}$ be ICS in IOCS algorithm and $m=1$. Assuming that EVs are released in $b$ distinct groups where arrival time of EVs in each group are the same, the IOCS is $b\left(1+\frac{p}{p-q} \frac{s}{s-1}\right)$-competitive with optimal offline solution, where $p$ is the station peak and $q=\max _{i} k_{i}, i=1, \ldots, n$.

\section{Simulation Results}

Simulation Setup and Overview: We consider charging scheduling of EVs during a period of 12 time slots of length 1 hour (e.g., from 08:00 to 20:00). We gathered information of 12 popular EV models in the market to use in the simulation. Each EV model is characterized by its battery capacity as shown in Table 3 and maximum charging rate. Tesla models can get charged with up to
TABLE 3: EV models and their battery capacity.

\begin{tabular}{|l|c|l|c|}
\hline Model & Battery & Model & Battery \\
\hline \hline Mitsubishi i-MiEV & $16 \mathrm{kWh}$ & Citroen C-Zero & $14 \mathrm{kWh}$ \\
\hline Peugeot iOn & $16 \mathrm{kWh}$ & Honda Clarity & $25.5 \mathrm{kWh}$ \\
\hline Hyundai Kona & $64 \mathrm{kWh}$ & Nissan LEAF & $40 \mathrm{kWh}$ \\
\hline Hyundai Ioniq & $28 \mathrm{kWh}$ & BMW i3 & $22 / 33 \mathrm{kWh}$ \\
\hline Tesla Model S/X & $60 / 100 \mathrm{kWh}$ & Kia Soul EV & $27 \mathrm{kWh}$ \\
\hline
\end{tabular}

TABLE 4: Acronyms for the algorithms

\begin{tabular}{|l|l|}
\hline Notation & Description \\
\hline \hline IOPT & Optimal value under integral revenue model \\
\hline ICS & $\begin{array}{l}\text { Proposed offline algorithm for SPAN under in- } \\
\text { tegral revenue model }\end{array}$ \\
\hline FCS & $\begin{array}{l}\text { Proposed optimal algorithm for SPAN under } \\
\text { fractional revenue model }\end{array}$ \\
\hline IOCS & $\begin{array}{l}\text { Proposed online algorithm for SPAN under in- } \\
\text { tegral revenue model }\end{array}$ \\
\hline FOCS & $\begin{array}{l}\text { Proposed online algorithm for SPAN under frac- } \\
\text { tional revenue model }\end{array}$ \\
\hline IOLP & $\begin{array}{l}\text { Algorithm in [7] (works under integral revenue } \\
\text { model) }\end{array}$ \\
\hline FOLP & $\begin{array}{l}\text { Adaptation of algorithm in [7] for fractional } \\
\text { revenue model }\end{array}$ \\
\hline GreedyRTL & $\begin{array}{l}\text { The algorithm in [35] for single station scenario } \\
\text { without global peak constraint (works under } \\
\text { integral revenue model) }\end{array}$ \\
\hline
\end{tabular}

$100 \mathrm{~kW}$ using Tesla super chargers. Also, all other models have rapid DC charging capability (up to $50 \mathrm{~kW}$ ) with CHAdeMO method. This setting for charging rates is in accordance with future DCFC systems that can have several fast DC chargers installed in the charging station. The peak intervals include 08:00-10:00, 12:00-14:00, and 18:00-20:00 according to NHTS survey [4], [41]. The probability of an EV's arrival during the peak hours is two times higher than the off-peak periods. Demands are uniform random values from $\left[\frac{1}{2} U_{i}, U_{i}\right]$ where $U_{i}$ is the battery capacity for EV $i$ in Table 3. The deadline of each EV is set according to slackness parameter $s$ (with default value of 1.2), its demand and maximum charging rate of the battery as $d_{i}=a_{i}+\left\lceil\frac{D_{i} s}{k_{i}}\right\rceil-1$. EVs are assigned to different CSs randomly and given as input to the algorithms. The willingness to pay by each user for one $\mathrm{kWh}$ of power is a random uniform number from interval $\left[\frac{1}{2} z, \frac{3}{2} z\right]$ where $z=\$ 0.11$ is national average price of electricity in the US [42]. In the simulation, the results are plotted with $95 \%$ confidence level and each point represents average result of 50 random scenarios. Table 4 explains the comparison algorithms including current implemented algorithm in Caltech ACN referred to as IOLP [7] and its adapted version for the fractional revenue model FOLP. The IOLP and FOLP algorithms runs as follows: at each time slot assume that there will be no further arrivals and solve the optimization problem (e.g., by commercial solvers) for the current set of EVs and their remaining demands. In Table 4, the letters " $\mathrm{I}$ ", " $\mathrm{F}$ " and "O" in front of the algorithms' name refer to integral, fractional and online types, respectively.

The measured performance metrics are total revenue, percentage of EVs that received all their demand, and total peak. To calculate optimal solution in integral revenue model, we used Gurobi solver [43].

Evaluation Based on Total Revenue: Fig. 2 depicts the comparison results based on total revenue under fractional and integral models while the total number of EVs increases 


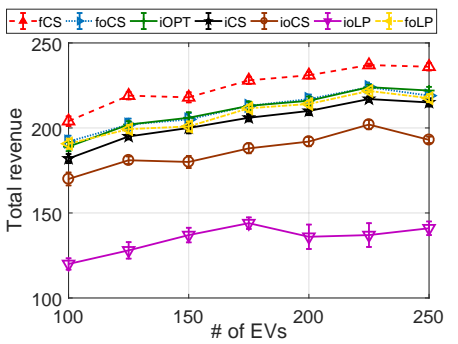

(a) $m=2$

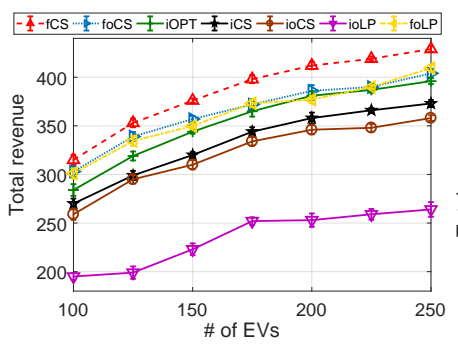

(b) $m=4$

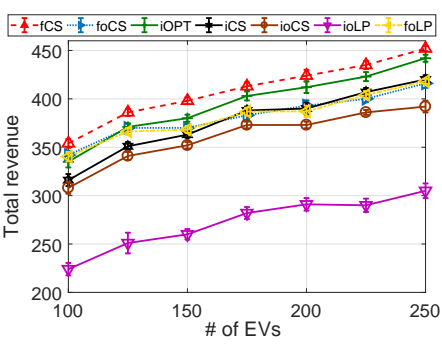

(c) $m=8$

Fig. 2: Comparison results for 2, 4, and 8 CSs for fractional and integral revenue models. In the algorithms' name, letters "o", " $\mathrm{f}$ " and " $\mathrm{i}$ " indicate online, fractional and integral, respectively. Note that a "non-optimal" fractional algorithm can potentially achieve better result than an "optimal" integral algorithm due to higher flexibility in power allocation.

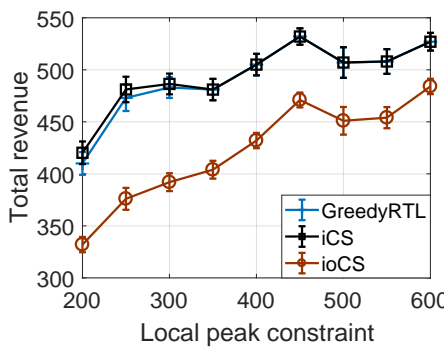

(a) Total revenue

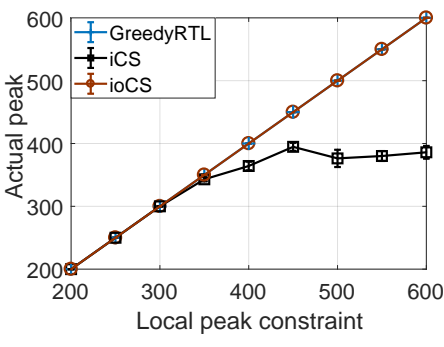

(b) Actual global peak

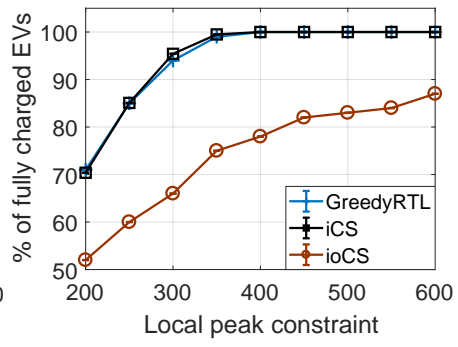

(c) \% of full charged EVs

Fig. 3: Comparison in terms of total revenue, actual peak, and percentage of fully charged EVs by varying local peak value.

from 100 to 250 for 2,4 , and 8 CSs. The local and global peak constraints are set to $50 \mathrm{kWh}$ and $200 \mathrm{kWh}$, respectively.

he proposed algorithms are compared to the optimal offline solution of integral model (IOPT) and IOLP. Note that comparison with offline optimal could be considered as a baseline comparison with the category of approximate offline algorithms in integral model [36].

Recall that FCS is optimal offline solution. The notable observations are as follows: (i) the general trend in scenario of Fig. 2 is that by increasing the number of EVs, total revenue increases. This is because with more number of EVs, the scheduler has more freedom to choose more valuable EVs. (ii) as explained in Section 4, under fractional charging model, better results are expected due to increased scheduling flexibility in CSs. According to the simulation data that we extracted from Fig. 2, the gain obtained by FCS and FOCS in Fig. 2 are respectively $12 \%$ and $10 \%$ more than the gain of the ICS and IOCS. (iii) In Fig. 2a and Fig. 2b, sum of the local peaks is less than or equal to the global peak while in Fig. $2 \mathrm{c}$ this sum is two times greater than the global peak. Consequently, the total revenue significantly improved when the number of CSs is increased from 2 to 4 while there is a slight improvement from 4 to 8 CSs as the global peak constraint prevents the algorithms from charging more EVs. (iv) in integral revenue model, the proposed IOCS algorithm acts significantly better than IOLP. In particular, IOCS improves IOLP by $38 \%, 36 \%$, and $32 \%$ for $m=2, m=4$ and $m=8$, respectively. In fractional revenue model, however, there is a very slight difference between FOCS and FOLP. (v) ICS approximates IOPT by $97 \%, 94 \%$, and $95 \%$ for $m=2, m=4$, and $m=8$, respectively. On average, IOCS is $90 \%$ of IOPT and, FOCS is $94 \%$ of its optimal offline solution, FCS. (vi) finally, the results depict that ICS achieves much better results in practice as compared to the theoretical approximation ratio that characterizes the performance in worst-case scenario.

Comparison Based on Actual Peak: The constraint set in the SPAN assures that any feasible solution respects the local and global peak constraints. An efficient scheduling algorithm may take a further step by not only satisfying the peak constraints but to further reduce the peak as much as possible. The proposed offline algorithms (i.e., ICS and FCS) apply valley-filling policy to reduce the peak. The online algorithms (i.e., IOCS and FOCS) do not apply the same policy as they do not have future knowledge to be able to balance allocated resources. To investigate the effect of employed valley-filling strategy, we conducted a set of simulations by varying local peak constraints. The results of ICS is compared to IOCS and GreedyRTL [35] where the latter is an approximation scheduling for single station scenario with EVs having same arrival time (see the explanations after formulating the SPAN in Section 3.2). The results are shown in Fig. 3. Along with total revenue in Fig. 3a, we also report total actual peak in Fig. 3b and percentage of fully charged EVs in Fig. 3c. In Fig. 3a, the results for ICS and GreedyRTL are almost identical while IOCS is $90 \%$ of the other two algorithms, on average. When $p_{j} \geq 400$, the total revenue for ICS and GreedyRTL does not increase in Fig. 3a and percentage of fully charged EVs is 100 for both algorithms according to Fig. 3c. From this point and onward, the scheduling is not challenging for offline methods to obtain optimal answer because of resource sufficiency. However, it is still a challenge to control total actual peak for the system. As a result of valley-filling policy, the value of actual peak of ICS in Fig. 3b remains almost unchanged for $p_{j} \geq 400$. IOCS and GreedyRTL, 
however, continuously increase the peak demand, since they are not using any peak shaving approach.

\section{Conclusion}

This paper proposed offline and online algorithms for the EV charging scheduling problem under fractional and integral revenue models in an adaptive charging network $(\mathrm{ACN})$. The problem is different, and more challenging than the existing single station EV charging scheduling problems since it requires respecting the aggregate peak charging demand of the ACN. As the notable contributions, our proposed online algorithm for fractional revenue model achieves constant competitive ratio of 2. Moreover, the offline integral algorithm achieves a theoretical bound on the optimality gap and approximates the optimum by $92 \%$, on average in experiments. As a future work, we plan to study the problem under posted pricing mechanism where the charging station publishes the unit price of the power (which can be varied over time) and the users can accept or reject the offer. Another interesting future direction is to tackle the EV charging scheduling in market-based scenarios where the prices change over time.

\section{REFERENCES}

[1] "https://www.bloomberg.com/news/articles/2017-0706/electric-cars-are-about-to-boost-global-power-demand-300fold," 2017.

[2] S. Zhao, X. Lin, and M. Chen, "Peak-minimizing online ev charging: Price-of-uncertainty and algorithm robustification," in Proc. of IEEE INFOCOM, 2015.

[3] J. C. Mukherjee and A. Gupta, "A review of charge scheduling of electric vehicles in smart grid," IEEE Systems Journal, vol. 9, no. 4, pp. 1541-1553, 2015.

[4] W. Tang, S. Bi, and Y. J. A. Zhang, "Online coordinated charging decision algorithm for electric vehicles without future information," IEEE Transactions on Smart Grid, vol. 5, no. 6, pp. 2810-2824, 2014.

[5] D. Wu, H. Zeng, C. Lu, and B. Boulet, "Two-stage energy management for office buildings with workplace ev charging and renewable energy," IEEE Transactions on Transportation Electrification, vol. 3, no. 1, pp. 225-237, 2017.

[6] G. Lee, T. Lee, Z. Low, S. H. Low, and C. Ortega, "Adaptive charging network for electric vehicles," in Proc. of IEEE GlobalSIP, 2016.

[7] Z. J. Lee, D. Chang, C. Jin, G. S. Lee, R. Lee, T. Lee, and S. H. Low, "Large-Scale Adaptive Electric Vehicle Charging," in IEEE International Conference on Communications, Control, and Computing Technologies for Smart Grids, Oct. 2018.

[8] C.-K. Wen, J.-C. Chen, J.-H. Teng, and P. Ting, "Decentralized plugin electric vehicle charging selection algorithm in power systems," IEEE Transactions on Smart Grid, vol. 3, no. 4, pp. 1779-1789, 2012.

[9] Z. Zheng and N. Shroff, "Online welfare maximization for electric vehicle charging with electricity cost," in Proc. of ACM e-energy, pp. 253-263, ACM, 2014.

[10] W. Tang and Y. J. A. Zhang, "A model predictive control approach for low-complexity electric vehicle charging scheduling: optimality and scalability," IEEE Transactions on Power Systems, vol. 32, no. 2, pp. 1050-1063, 2017.

[11] S. Chen and L. Tong, "iEMS for large scale charging of electric vehicles: Architecture and optimal online scheduling," in Proc. of SmartGridComm, 2012

[12] Q. Xiang, F. Kong, X. Liu, X. Chen, L. Kong, and L. Rao, "Auc2charge: An online auction framework for eectric vehicle park-and-charge," in Proc. of ACM e-energy, pp. 151-160, ACM, 2015.

[13] V. Robu, E. H. Gerding, S. Stein, D. C. Parkes, A. Rogers, and N. R. Jennings, "An online mechanism for multi-unit demand and its application to plug-in hybrid electric vehicle charging," Journal of Artificial Intelligence Research, vol. 48, pp. 175-230, 2013.
[14] "http://www.pge.com/nots/rates/tariffs/rateinfo.shtml," 2018.

[15] Y. Zhang, M. Hajiesmaili, S. Cai, M. Chen, and Q. Zhu, "Peakaware online economic dispatching for microgrids," IEEE Transactions on Smart Grid, vol. 9, no. 1, pp. 323-335, 2018.

[16] A. Borodin and R. El-Yaniv, Online computation and competitive analysis. cambridge university press, 2005.

[17] V. V. Vazirani, Approximation algorithms. Springer Science \& Business Media, 2013.

[18] R. D. Carr, L. K. Fleischer, V. J. Leung, and C. A. Phillips, "Strengthening integrality gaps for capacitated network design and covering problems," tech. rep., Sandia National Labs., Albuquerque, NM (US); Sandia National Labs., Livermore, CA (US), 1999.

[19] B. Alinia, M. S. Talebi, M. H. Hajiesmaili, A. Yekkehkhany, and N. Crespi, "Competitive online scheduling algorithms with applications in deadline-constrained EV charging," in 2018 IEEE/ACM 26th International Symposium on Quality of Service (IWQoS), pp. 110, IEEE, 2018.

[20] E. S. Rigas, S. D. Ramchurn, N. Bassiliades, and G. Koutitas, "Congestion management for urban ev charging systems," in Proc. of IEEE SmartGridComm, pp. 121-126, 2013.

[21] J. Hu, S. You, M. Lind, and J. Østergaard, "Coordinated charging of electric vehicles for congestion prevention in the distribution grid," IEEE Transactions on Smart Grid, vol. 5, no. 2, pp. 703-711, 2013.

[22] P. M. de Quevedo, G. Muñoz-Delgado, and J. Contreras, "Impact of electric vehicles on the expansion planning of distribution systems considering renewable energy, storage and charging stations," IEEE Transactions on Smart Grid, 2017.

[23] M. Shafie-Khah, P. Siano, D. Z. Fitiwi, N. Mahmoudi, and J. P. Catalão, "An innovative two-level model for electric vehicle parking lots in distribution systems with renewable energy," IEEE Transactions on Smart Grid, vol. 9, no. 2, pp. 1506-1520, 2018.

[24] S. Huang, Q. Wu, S. S. Oren, R. Li, and Z. Liu, "Distribution locational marginal pricing through quadratic programming for congestion management in distribution networks," IEEE Transactions on Power Systems, vol. 30, no. 4, pp. 2170-2178, 2014.

[25] Y. He, B. Venkatesh, and L. Guan, "Optimal scheduling for charging and discharging of electric vehicles," IEEE transactions on smart grid, vol. 3, no. 3, pp. 1095-1105, 2012.

[26] A. Malhotra, G. Binetti, A. Davoudi, and I. D. Schizas, "Distributed power profile tracking for heterogeneous charging of electric vehicles," IEEE Transactions on Smart Grid, vol. 8, no. 5, pp. 2090-2099, 2017.

[27] M. Moradijoz, M. P. Moghaddam, M. Haghifam, and E. Alishahi, "A multi-objective optimization problem for allocating parking lots in a distribution network," International Journal of Electrical Power \& Energy Systems, vol. 46, pp. 115-122, 2013.

[28] D. Wang, X. Guan, J. Wu, P. Li, P. Zan, and H. Xu, "Integrated energy exchange scheduling for multimicrogrid system with electric vehicles," IEEE Transactions on Smart Grid, vol. 7, no. 4, pp. 17621774, 2016.

[29] M. Zeng, S. Leng, Y. Zhang, and J. He, "Qoe-aware power management in vehicle-to-grid networks: a matching-theoretic approach," early access, IEEE Transactions on Smart Grid, 2017.

[30] M. F. Shaaban, M. Ismail, E. F. El-Saadany, and W. Zhuang, "Realtime pev charging/discharging coordination in smart distribution systems," IEEE Transactions on Smart Grid, vol. 5, no. 4, pp. 17971807, 2014.

[31] R. Lee, M. H. Hajiesmaili, and J. Li, "Learning-assisted competitive algorithms for peak-aware energy scheduling," arXiv preprint arXiv:1911.07972, 2019.

[32] M. H. Hajiesmaili, C.-K. Chau, M. Chen, and L. Huang, "Online microgrid energy generation scheduling revisited: The benefits of randomization and interval prediction," in Proc. of ACM e-energy, p. 1, ACM, 2016.

[33] M. H. Hajiesmaili, M. Chen, E. Mallada, and C.-K. Chau, "Crowdsourced storage-assisted demand response in microgrids," in Proc. of ACM eEnergy, pp. 91-100, ACM, 2017.

[34] B. Alinia, M. Hajiesmaili, and N. Crespi, "Online EV charging scheduling with on-arrival commitment," in submission, 2018.

[35] N. Jain, I. Menache, J. S. Naor, and J. Yaniv, "Near-optimal scheduling mechanisms for deadline-sensitive jobs in large computing clusters," ACM Transactions on Parallel Computing, vol. 2, no. 1, p. 3, 2015.

[36] L. Yao, W. H. Lim, and T. S. Tsai, "A real-time charging scheme for demand response in electric vehicle parking station," IEEE Transactions on Smart Grid, vol. 8, no. 1, pp. 52-62, 2016. 
[37] E.-C. Chang and C. Yap, "Competitive on-line scheduling with level of service," Journal of Scheduling, vol. 6, no. 3, pp. 251-267, 2003.

[38] Z. Zheng and N. B. Shroff, "Online multi-resource allocation for deadline sensitive jobs with partial values in the cloud," in IEEE INFOCOM 2016-The 35th Annual IEEE International Conference on Computer Communications, pp. 1-9, IEEE, 2016.

[39] M. M. de Weerdt, M. Albert, V. Conitzer, and K. v. d. Linden, "Complexity of scheduling charging in the smart grid," in Proceedings of the 17th International Conference on Autonomous Agents and MultiAgent Systems, pp. 1924-1926, International Foundation for Autonomous Agents and Multiagent Systems, 2018.

[40] B. Lucier, I. Menache, J. S. Naor, and J. Yaniv, "Efficient online scheduling for deadline-sensitive jobs," in Proceedings of the twentyfifth annual ACM symposium on Parallelism in algorithms and architectures, pp. 305-314, ACM, 2013.

[41] A. Santos, N. McGuckin, H. Y. Nakamoto, D. Gray, and S. Liss, "Summary of travel trends: 2009 national household travel survey," tech. rep., 2011.

[42] "https://www.eia.gov/electricity/state/."

[43] "Gurobi optimizer 5.0," Gurobi: http://www.gurobi.com, 2013.

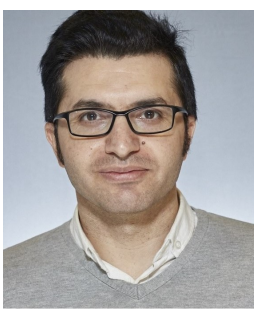

Bahram Alinia is an $R \& D$ software engineer in France since 2018. He received a joint PhD degree from Institute Telecom SudParis/ParisSorbonne University (UPMC) in 2018, a M.Sc. degree of IT engineering from university of Tehran, Iran, in 2012, and a B.Sc. degree of computer science from University of Tabriz, Tabriz, Iran in 2008.

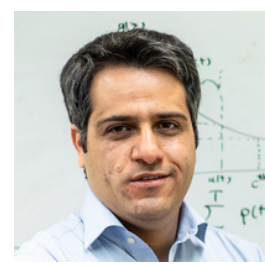

Mohammad H. Hajiesmaili is a Research Assistant Professor in the College of Information and Computer Sciences at the University of Massachusetts Amherst. Before joining UMass, Mohammad was a postdoctoral fellow with the Johns Hopkins University, from 2017 to 2018, and with the Chinese University of Hong Kong, from 2015 to 2016. He received his Ph.D. and M.Sc. degrees from the University of Tehran, and his B.Sc. degree from Sharif University of Technology.

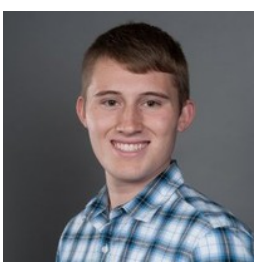

Zachary J. Lee is a Ph.D. student in Electrical Engineering at the California Institute of Technology (Caltech). He received his M.S. in Electrical Engineering from Caltech in 2018 and his B.S.Eng. in Electrical Engineering from John Brown University in 2016. He is a recipient of the National Science Foundation Graduate Research Fellowship and Resnick Sustainability Institute Graduate Fellowship. His research is centered on smart electric vehicle charging, where computer science, and energy.

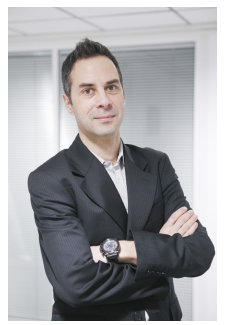

Prof. Noël Crespi holds Masters degrees from the Universities of Orsay (Paris 11) and Kent (UK), a diplome d'ingénieur from Telecom ParisTech, a Ph.D and an Habilitation from UPMC (Paris-Sorbonne University). From 1993 he worked at CLIP, Bouygues Telecom and then at Orange Labs in 1995. He took leading roles in the creation of new services with the successful conception and launch of Orange prepaid service, and in standardisation (from rapporteurship of IN standard to coordination of all mobile standards activities for Orange). In 1999, he joined Nortel Networks as telephony program manager, architecting core network products for EMEA region. He joined Institut Mines-Telecom in 2002 and is currently professor and Program Director, leading the Service Architecture Lab. He coordinates the standardisation activities for Institut Mines-Telecom at ITU-T and ETSI. He is also an adjunct professor at KAIST (South Korea), an affiliate professor at Concordia University (Canada), and gest researcher at the University of Goettingen (Germany). He is the scientific director the French-Korean laboratory ILLUMINE. His current research interests are in Data Analytics, Internet of Things and Softwarisation. http://noelcrespi.wp.tem-tsp.eu/

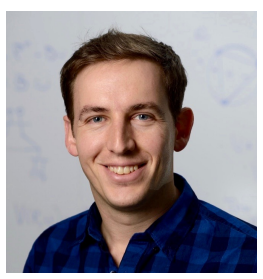

Enrique Mallada is an assistant professor of electrical and computer engineering at Johns Hopkins University since 2016. Before joining Hopkins, he was a post-doctoral fellow at the Center for the Mathematics of Information at the California Institute of Technology from 2014 to 2016. He received his ingeniero en telecomunicaciones (telecommunications engineering) degree from Universidad ORT, Uruguay, in 2005 and his Ph.D. degree in electrical and computer engineering with a minor in applied mathematics from Cornell University in 2014. Dr. Mallada was awarded the NSF CAREER award in 2018, the ECE Director's Ph.D. Thesis Research Award for his dissertation in 2014, the Cornell University's Jacobs Fellowship in 2011 and the Organization of American States scholarship from 2008 to 2010 . His research interests lie in the areas of control, networked dynamics, and optimization, with applications to engineering networks such as power systems and the Internet. 